\title{
Stochastic convergence analysis of cubature Kalman filter with intermittent observations
}

\author{
SHI Jie ${ }^{1,2}$, QI Guoqing ${ }^{1, *}$, LI Yinya ${ }^{1}$, and SHENG Andong ${ }^{1}$ \\ 1. School of Automation, Nanjing University of Science and Technology, Nanjing 210094, China; \\ 2. NARI Group Corporation/State Grid Electric Power Research Institute, Nanjing 211000, China
}

\begin{abstract}
The stochastic convergence of the cubature Kalman filter with intermittent observations (CKFI) for general nonlinear stochastic systems is investigated. The Bernoulli distributed random variable is employed to describe the phenomenon of intermittent observations. According to the cubature sample principle, the estimation error and the error covariance matrix (ECM) of CKFI are derived by Taylor series expansion, respectively. Afterwards, it is theoretically proved that the ECM will be bounded if the observation arrival probability exceeds a critical minimum observation arrival probability. Meanwhile, under proper assumption corresponding with real engineering situations, the stochastic stability of the estimation error can be guaranteed when the initial estimation error and the stochastic noise terms are sufficiently small. The theoretical conclusions are verified by numerical simulations for two illustrative examples; also by evaluating the tracking performance of the optical-electric target tracking system implemented by $\mathrm{CKFI}$ and unscented Kalman filter with intermittent observations (UKFI) separately, it is demonstrated that the proposed CKFI slightly outperforms the UKFI with respect to tracking accuracy as well as real time performance.
\end{abstract}

Keywords: cubature Kalman filter (CKF), intermittent observation, estimation error, stochastic stability.

DOI: $10.21629 /$ JSEE.2018.04.17

\section{Introduction}

The nonlinear state estimation problem is a hot research topic that has attracted considerable interest during the past decades. A great deal of suboptimal approaches have been proposed to deal with the nonlinear filtering problem, such as extended Kalman filter (EKF) [1], unscented Kalman filter (UKF) [2,3], and particle filter (PF) [4,5]. These methods mentioned above have been successfully adopted in many applications, including simultaneous localization and mapping (SLAM) problem [6], state estimation in power systems [7], maneuvering target tracking

\footnotetext{
Manuscript received March 07, 2017.

*Corresponding author.

This work was supported by the National Natural Science Foundation of China (61104186; 61273076).
}

with a multistatic radar system [8], and so forth.

In 2010, a new nonlinear filter called cubature Kalman filter (CKF) was proposed by Arasaratnam and Haykin $[9,10]$. The CKF was developed on the basis of the spherical-radial cubature rule that is a useful way to numerically compute multivariate moment integrals encountered in the nonlinear Bayesian filtering problems [11]. Similar to the UKF, it is also not necessary to compute the Jacobian matrix which is the basis of EKF. The CKF only propagates $2 n$ equal-weighted cubature points through a nonlinear function to approximate the mean and covariance of state and measurement vector [12]. Meanwhile, the sigma points of UKF have some adjustment parameters that should be set reasonably while the weights of cubature points of CKF are fixed. The CKF can achieve better estimation performance compared to the EKF and UKF, especially for high-dimensional nonlinear filtering problems, e.g., state estimation of chemical reaction [13], spacecraft attitude and position estimation [14], maneuvering target tracking [15], bearings-only tracking [16], GPS/IMU tightly-coupled navigation system [17], etc. Most importantly, in $[18,19]$, by the linearization of the nonlinear model, the convergence analysis and the estimation performance of CKF for nonlinear discrete-time systems were investigated.

In practical engineering, however, the estimator may be implemented under the condition that the observations contain missing measurements. Generally, the reasons for the phenomenon of intermittent observations can be classified into the following two aspects. For one thing, it is quite common in practice that the observations may not be acquired caused by the high maneuverability of the tracked target, intermittent sensor failures or accidental loss of some collected data [20,21]. For another, the observations could be lost or delayed due to the transmission failure and signal fluctuation of the communication channel between the sensor and estimator $[22,23]$. Therefore, the effect of 
intermittent observations in practical engineering applications must be highly considered. Early interesting results on linear estimation with intermittent observations can be traced back to [24]. In [24], the arrival of observations was modeled as an independent, identically distributed (i.i.d) Bernoulli process and the existence of a corresponding critical probability has been shown to guarantee the boundedness of the error covariance matrix (ECM). Afterward, a two state Markov chain was introduced to model the arrival of observations in [25], where the sufficient condition for the boundedness of peak ECM was presented. Recently, a necessary condition and a sufficient condition were derived for the boundedness of the expected value of the ECM of the Kalman filter with intermittent observations [26].

On the other hand, in the case of nonlinear filtering with intermittent observations, considerable publications can be retrieved in the literature (see, e.g. [27-31] and the references therein). The stochastic stability problems of the EKF-based estimation methods for general nonlinear systems with intermittent observations were investigated in [27,28]. In [27], under appropriate assumptions, a critical probability also existed to ensure the boundedness of the prediction ECM, and the estimation error was exponentially bounded in mean square if the ECM and the initial estimation error remain bounded. The stochastic stability analysis of a new extended filtering (EF) subject to measurement packet losses was presented in [28]. Similar to the case in [27], conditions for guaranteeing the stochastic stability of the UKF with intermittent observations (UKFI) have been developed in [29,30]. In addition, the peak ECM stability of UKFI was studied in [31], where the arrival of observations was modeled as a two state timehomogeneous Markov process.

Above all, the stochastic convergence analysis of CKF with complete observations has been conducted in [18]. In contrast to the theoretical research of [18], we focus on the CKF-based state estimation problem for nonlinear systems with intermittent observations, which often occur in practical applications such as target tracking. The main contribution of this work can be highlighted as follows:

(i) The Taylor series expansion is adopted to derive the estimation error and ECM of the CKF with intermittent observations (CKFI), which enables us to analyze the stochastic convergence of CKFI.

(ii) The conditions are provided regarding the boundedness of ECM and the stochastic stability of estimation error, respectively.

(iii) To compare the estimation performances of the proposed CKFI and UKFI, both algorithms are implemented in the optic-electric target tracking system.

The remainder of this paper is organized as follows:
Section 2 establishes the filtering framework of CKFI. The estimation errors and ECM of CKFI are derived in Section 3. The theoretical results on the boundedness of ECM and the stochastic stability of estimation error are provided in Section 4 and Section 5, respectively. Section 6 presents two illustrated examples and the optic-electric target tracking system implemented by CKFI and UKFI. A summary of conclusion is given in Section 7 .

The notation used in this article is fairly standard. $\mathbf{R}^{n}$ denotes the $n$ dimensional Euclidean space, $\mathbf{R}^{m \times n}$ is a set of real matrices with dimension $m \times n$, the superscript "T" stands for matrix transposition. $\boldsymbol{I}_{n}$ denotes the identity matrix with dimension $n$, and $\boldsymbol{I}$ denotes the identity matrix with suitable dimension. The norm of vector $\|\boldsymbol{x}\|$ represents the Euclidean norm, the norm of matrix $\|\boldsymbol{X}\|$ represents the spectral norm. The $\mathrm{E}(\boldsymbol{x})$ stands for the expectation value of $\boldsymbol{x}$.

\section{Problem formulation}

We consider the nonlinear discrete-time stochastic system with intermittent observations [24]:

$$
\left\{\begin{array}{l}
\boldsymbol{X}_{k}=\boldsymbol{f}\left(\boldsymbol{X}_{k-1}\right)+\boldsymbol{w}_{k-1} \\
\boldsymbol{Z}_{k}=d_{k} \boldsymbol{h}\left(\boldsymbol{X}_{k}\right)+\boldsymbol{v}_{k}
\end{array}\right.
$$

where $\boldsymbol{X}_{k} \in \mathbf{R}^{n_{x}}$ is the state vector, $\boldsymbol{Z}_{k} \in \mathbf{R}^{n_{z}}$ is the measurement vector. $\boldsymbol{w}_{k}$ and $\boldsymbol{v}_{k}$ are unrelated zero-mean white Gaussian vectors with covariance $\boldsymbol{Q}_{k}$ and $\boldsymbol{R}_{k}$, respectively. We use $d_{k}$ to indicate whether the observation $Z_{k}$ is received. $d_{k}$ is a scalar binary Bernoulli distributed random variable taking values on 0 and 1 , and the random variable $d_{k}$ can be characterized by probability $\lambda$ with $\operatorname{Prob}\left(d_{k}=1\right)=\lambda$ and $\operatorname{Prob}\left(d_{k}=0\right)=1-\lambda$. The observation noise $\boldsymbol{v}_{k}$ is defined as

$$
p\left(\boldsymbol{v}_{k} \mid d_{k}\right) \sim \begin{cases}N\left(0, \boldsymbol{R}_{k}\right), & d_{k}=1 \\ N\left(0, \sigma^{2} \boldsymbol{I}\right), & d_{k}=0\end{cases}
$$

the absence of observation corresponds to the limiting case of $\sigma^{2} \rightarrow \infty$.

Assume the posterior density function $p\left(\boldsymbol{X}_{k-1} \mid \boldsymbol{Z}_{1: k-1}\right.$, $\left.d_{1: k-1}\right) \sim N\left(\boldsymbol{X}_{k-1} ; \hat{\boldsymbol{X}}_{k-1 \mid k-1}, \hat{\boldsymbol{P}}_{k-1 \mid k-1}\right)$ is known at time step $k-1$. The procedure for implementing the CKFI can be summarized as

Step 1 Evaluate cubature points, $i=1,2, \ldots, m$

$$
\chi_{i, k-1 \mid k-1}=\sqrt{\hat{\boldsymbol{P}}_{k-1 \mid k-1}} \eta_{i}+\hat{\boldsymbol{X}}_{k-1 \mid k-1}
$$

where $m=2 n_{x}, n_{x}$ is the dimension of state vector. $\sqrt{\hat{\boldsymbol{P}}_{k-1 \mid k-1}}$ is the matrix square root of $\hat{\boldsymbol{P}}_{k-1 \mid k-1}$, and $\eta_{i}=\sqrt{m / 2}[1]_{i}$ is the $i$ th element of the following vector set: 


$$
\sqrt{\frac{m}{2}}\{\underbrace{\left(\left(\begin{array}{c}
1 \\
0 \\
\vdots \\
0
\end{array}\right),\left(\begin{array}{c}
0 \\
1 \\
\vdots \\
0
\end{array}\right), \ldots,\left(\begin{array}{c}
0 \\
0 \\
\vdots \\
1
\end{array}\right),\left(\begin{array}{c}
-1 \\
0 \\
\vdots \\
0
\end{array}\right),\left(\begin{array}{c}
0 \\
-1 \\
\vdots \\
0
\end{array}\right), \ldots,\left(\begin{array}{c}
0 \\
0 \\
\vdots \\
-1
\end{array}\right)\right\}}_{m} .
$$

Step 2 Time update

$$
\left\{\begin{array}{l}
\boldsymbol{X}_{i, k \mid k-1}^{*}=\boldsymbol{f}\left(\boldsymbol{\chi}_{i, k-1 \mid k-1}\right) \\
\hat{\boldsymbol{X}}_{k \mid k-1}=\sum_{i=1}^{m} w_{i} \boldsymbol{X}_{i, k \mid k-1}^{*} \\
\hat{\boldsymbol{P}}_{k \mid k-1}=\sum_{i=1}^{m} w_{i} \boldsymbol{X}_{i, k \mid k-1}^{*} \boldsymbol{X}_{i, k \mid k-1}^{* \mathrm{~T}}- \\
\quad \hat{\boldsymbol{X}}_{k \mid k-1} \hat{\boldsymbol{X}}_{k \mid k-1}^{\mathrm{T}}+\boldsymbol{Q}_{k-1}
\end{array}\right.
$$

where $w_{i}=1 / m \cdot \hat{\boldsymbol{X}}_{k \mid k-1}$ is the state prediction, and $\hat{\boldsymbol{P}}_{k \mid k-1}$ is the state error covariance prediction.

Step 3 Measurement update

$$
\left\{\begin{array}{l}
\boldsymbol{X}_{i, k \mid k-1}=\sqrt{\hat{\boldsymbol{P}}_{k \mid k-1}} \eta_{i}+\hat{\boldsymbol{X}}_{k \mid k-1} \\
\boldsymbol{Z}_{i, k \mid k-1}=\boldsymbol{h}\left(\boldsymbol{X}_{i, k \mid k-1}\right) \\
\hat{\boldsymbol{Z}}_{k \mid k-1}=\sum_{i=1}^{m} w_{i} \boldsymbol{Z}_{i, k \mid k-1} \\
\hat{\boldsymbol{P}}_{z z, k \mid k-1}=\sum_{i=1}^{m} w_{i} \boldsymbol{Z}_{i, k \mid k-1} \boldsymbol{Z}_{i, k \mid k-1}^{\mathrm{T}}- \\
\hat{\boldsymbol{Z}}_{k \mid k-1} \hat{\boldsymbol{Z}}_{k \mid k-1}^{\mathrm{T}}+d_{k} \boldsymbol{R}_{k}+\left(1-d_{k}\right) \sigma^{2} \boldsymbol{I} \\
\hat{\boldsymbol{P}}_{x z, k \mid k-1}=\sum_{i=1}^{m} w_{i} \boldsymbol{X}_{i, k \mid k-1} \boldsymbol{Z}_{i, k \mid k-1}^{\mathrm{T}}-\hat{\boldsymbol{X}}_{k \mid k-1} \hat{\boldsymbol{Z}}_{k \mid k-1}^{\mathrm{T}}
\end{array}\right.
$$

where $\hat{Z}_{k \mid k-1}$ is the observation predictive estimation. $\hat{\boldsymbol{P}}_{z z, k \mid k-1}$ is the observation predictive error covariance and $\hat{\boldsymbol{P}}_{x z, k \mid k-1}$ is the cross-correlation covariance.

$$
\begin{aligned}
& \hat{\boldsymbol{X}}_{k \mid k}=\hat{\boldsymbol{X}}_{k \mid k-1}+\hat{\boldsymbol{P}}_{x z, k \mid k-1}\left[\sum_{i=1}^{m} w_{i} \boldsymbol{Z}_{i, k \mid k-1} \boldsymbol{Z}_{i, k \mid k-1}^{\mathrm{T}}-\right. \\
& \left.\hat{\boldsymbol{Z}}_{k \mid k-1} \hat{\boldsymbol{Z}}_{k \mid k-1}^{\mathrm{T}}+d_{k} \boldsymbol{R}_{k}+\left(1-d_{k}\right) \sigma^{2} \boldsymbol{I}\right]^{-1}\left(\boldsymbol{Z}_{k}-\hat{\boldsymbol{Z}}_{k \mid k-1}\right)
\end{aligned}
$$

$$
\begin{aligned}
& \hat{\boldsymbol{P}}_{k \mid k}=\hat{\boldsymbol{P}}_{k \mid k-1}-\hat{\boldsymbol{P}}_{x z, k \mid k-1}\left[\sum_{i=1}^{m} w_{i} \boldsymbol{Z}_{i, k \mid k-1} \boldsymbol{Z}_{i, k \mid k-1}^{\mathrm{T}}-\right. \\
& \left.\hat{\boldsymbol{Z}}_{k \mid k-1} \hat{\boldsymbol{Z}}_{k \mid k-1}^{\mathrm{T}}+d_{k} \boldsymbol{R}_{k}+\left(1-d_{k}\right) \sigma^{2} \boldsymbol{I}\right]^{-1} \hat{\boldsymbol{P}}_{x z, k \mid k-1}^{\mathrm{T}} \text { (7) }
\end{aligned}
$$

Taking the limit with $\sigma^{2} \rightarrow \infty$, it can be obtained that

$$
\left\{\begin{array}{l}
\hat{\boldsymbol{X}}_{k \mid k}=\hat{\boldsymbol{X}}_{k \mid k-1}+d_{k} \boldsymbol{K}_{k}\left(\boldsymbol{Z}_{k}-\hat{\boldsymbol{Z}}_{k \mid k-1}\right) \\
\hat{\boldsymbol{P}}_{k \mid k}=\hat{\boldsymbol{P}}_{k \mid k-1}-d_{k} \boldsymbol{K}_{k} \hat{\boldsymbol{P}}_{x z, k \mid k-1}^{\mathrm{T}}
\end{array}\right.
$$

where

$$
\begin{gathered}
\boldsymbol{K}_{k}=\hat{\boldsymbol{P}}_{x z, k \mid k-1}\left[\sum_{i=1}^{m} w_{i} \boldsymbol{Z}_{i, k \mid k-1} \boldsymbol{Z}_{i, k \mid k-1}^{\mathrm{T}}-\right. \\
\left.\hat{\boldsymbol{Z}}_{k \mid k-1} \hat{\boldsymbol{Z}}_{k \mid k-1}^{\mathrm{T}}+\boldsymbol{R}_{k}\right]^{-1} .
\end{gathered}
$$

Step 4 Repeat Steps 1-3 for the next sample.

\section{Estimation error and ECM of CKFI}

Define the estimation error $\tilde{\boldsymbol{X}}_{k+1 \mid k+1}$, state prediction error $\tilde{\boldsymbol{X}}_{k+1 \mid k}$ and observation prediction error $\tilde{\boldsymbol{Z}}_{k+1 \mid k}$ as

$$
\left\{\begin{array}{l}
\tilde{\boldsymbol{X}}_{k+1 \mid k+1}=\boldsymbol{X}_{k+1}-\hat{\boldsymbol{X}}_{k+1 \mid k+1} \\
\tilde{\boldsymbol{X}}_{k+1 \mid k}=\boldsymbol{X}_{k+1}-\hat{\boldsymbol{X}}_{k+1 \mid k} \\
\tilde{\boldsymbol{Z}}_{k+1 \mid k}=\boldsymbol{Z}_{k+1}-\hat{\boldsymbol{Z}}_{k+1 \mid k}
\end{array} .\right.
$$

Expending $\boldsymbol{X}_{k+1}$ by Taylor series about $\hat{\boldsymbol{X}}_{k \mid k}$ yields

$$
\begin{gathered}
\boldsymbol{X}_{k+1}=\boldsymbol{f}\left(\hat{\boldsymbol{X}}_{k \mid k}\right)+\nabla_{\tilde{\boldsymbol{X}}_{k \mid k}} \boldsymbol{f}\left(\hat{\boldsymbol{X}}_{k \mid k}\right)+ \\
\frac{1}{2 !} \nabla_{\tilde{\boldsymbol{X}}_{k \mid k}}^{2} \boldsymbol{f}\left(\hat{\boldsymbol{X}}_{k \mid k}\right)+\cdots+\boldsymbol{w}_{k}
\end{gathered}
$$

where $\nabla_{\tilde{\boldsymbol{X}}_{k \mid k}}^{i} \boldsymbol{f}\left(\hat{\boldsymbol{X}}_{k \mid k}\right)=\left.\left(\sum_{j=1}^{n_{x}} \tilde{x}_{j} \frac{\partial}{\partial x_{j}}\right)^{i} \boldsymbol{f}\left(\boldsymbol{X}_{k}\right)\right|_{\boldsymbol{X}_{k}=\hat{\boldsymbol{X}}_{k \mid k}}$, $x_{j}$ denotes the $j$ th element of $X . \nabla$ represents the differential of $f(X)$.

The sampling cubature points can be represented as

$$
\left\{\begin{array}{l}
\boldsymbol{\zeta}_{i, k}=\hat{\boldsymbol{X}}_{k \mid k}+\sqrt{n_{x} \hat{\boldsymbol{P}}_{k \mid k}}[1]_{i} \\
\boldsymbol{\zeta}_{i+n_{x}, k}=\hat{\boldsymbol{X}}_{k \mid k}-\sqrt{n_{x} \hat{\boldsymbol{P}}_{k \mid k}}[1]_{i}
\end{array}\right.
$$

where $i=1, \ldots, n_{x}$. Denote $\boldsymbol{\Delta}_{i, k}=\boldsymbol{\zeta}_{i, k}-\hat{\boldsymbol{X}}_{k \mid k}$. By using cubature transformation, we expend $\hat{\boldsymbol{X}}_{k+1 \mid k}$ by Taylor series [19]:

$$
\begin{gathered}
\hat{\boldsymbol{X}}_{k+1 \mid k}=\boldsymbol{f}\left(\hat{\boldsymbol{X}}_{k \mid k}\right)+\nabla^{\mathrm{T}} \hat{\boldsymbol{P}}_{k \mid k} \nabla \boldsymbol{f}\left(\hat{\boldsymbol{X}}_{k \mid k}\right) / 2 !+ \\
\frac{1}{n_{x}} \sum_{i=1}^{n_{x}}\left[\nabla_{\boldsymbol{\Delta}_{i, k}}^{4} \boldsymbol{f}\left(\hat{\boldsymbol{X}}_{k \mid k}\right) / 4 !+\nabla_{\boldsymbol{\Delta}_{i, k}}^{6} \boldsymbol{f}\left(\hat{\boldsymbol{X}}_{k \mid k}\right) / 6 !+\cdots\right] .
\end{gathered}
$$


Combing (10) and (12) we establish that

$$
\tilde{\boldsymbol{X}}_{k+1 \mid k}=\boldsymbol{F}_{k} \tilde{\boldsymbol{X}}_{k \mid k}+\boldsymbol{w}_{k}+\gamma\left(\hat{\boldsymbol{X}}_{k \mid k}, \boldsymbol{X}_{k}\right)
$$

where $\boldsymbol{F}_{k}=\left.[\partial \boldsymbol{f}(\boldsymbol{X}) / \partial \boldsymbol{X}]\right|_{\boldsymbol{X}=\hat{\boldsymbol{X}}_{k \mid k}}, \boldsymbol{\gamma}\left(\hat{\boldsymbol{X}}_{k \mid k}, \boldsymbol{X}_{k}\right)$ is the three and higher order residual for the Taylor polynomial of $f(X)$. An instrumental diagonal matrix $\boldsymbol{A}_{k}=$ $\operatorname{diag}\left\{a_{1, k}, \ldots, a_{n_{x}, k}\right\}$ is introduced to model the highorder residual. Then

$$
\tilde{\boldsymbol{X}}_{k+1 \mid k}=\boldsymbol{A}_{k} \boldsymbol{F}_{k} \tilde{\boldsymbol{X}}_{k \mid k}+\boldsymbol{w}_{k} .
$$

We denote $\boldsymbol{\sigma}_{i, k+1}=\boldsymbol{\pi}_{i, k+1}-\hat{\boldsymbol{X}}_{k+1 \mid k}$, where the cubature points are calculated as

$$
\left\{\begin{array}{l}
\boldsymbol{\pi}_{i, k+1}=\hat{\boldsymbol{X}}_{k+1 \mid k}+\sqrt{n_{x} \hat{\boldsymbol{P}}_{k+1 \mid k}}[1]_{i} \\
\boldsymbol{\pi}_{i+n_{x}, k+1}=\hat{\boldsymbol{X}}_{k+1 \mid k}-\sqrt{n_{x} \hat{\boldsymbol{P}}_{k+1 \mid k}}[1]_{i}
\end{array}\right.
$$

where $i=1, \ldots, n_{x}$. Similar to the calculating of state prediction error $\tilde{\boldsymbol{X}}_{k+1 \mid k}$, it can be derived that

$$
\begin{gathered}
\boldsymbol{Z}_{k+1}=\boldsymbol{h}\left(\hat{\boldsymbol{X}}_{k+1 \mid k}\right)+\nabla_{\tilde{\boldsymbol{X}}_{k+1 \mid k}} \boldsymbol{h}\left(\hat{\boldsymbol{X}}_{k+1 \mid k}\right)+ \\
\frac{1}{2 !} \nabla_{\tilde{\boldsymbol{X}}_{k+1 \mid k}}^{2} \boldsymbol{h}\left(\hat{\boldsymbol{X}}_{k+1 \mid k}\right)+\cdots+\boldsymbol{v}_{k+1} \\
\hat{\boldsymbol{Z}}_{k+1 \mid k}=\frac{1}{2 n_{x}}\left[\sum_{i=1}^{n_{x}} \boldsymbol{h}\left(\boldsymbol{\pi}_{i, k+1}\right)+\sum_{i=1}^{n_{x}} \boldsymbol{h}\left(\boldsymbol{\pi}_{i+n_{x}, k+1}\right)\right]= \\
\boldsymbol{h}\left(\hat{\boldsymbol{X}}_{k+1 \mid k}\right)+\nabla^{\mathrm{T}} \hat{\boldsymbol{P}}_{k+1 \mid k} \nabla \boldsymbol{h}\left(\hat{\boldsymbol{X}}_{k+1 \mid k}\right) / 2 !+ \\
\frac{1}{n_{x}} \sum_{i=1}^{n_{x}}\left[\frac{\nabla_{\sigma_{i, k+1}}^{4} \boldsymbol{h}\left(\hat{\boldsymbol{X}}_{k+1 \mid k}\right)}{4 !}+\frac{\nabla_{\sigma_{i, k+1}}^{6} \boldsymbol{h}\left(\hat{\boldsymbol{X}}_{k+1 \mid k}\right)}{6 !}+\cdots\right] .
\end{gathered}
$$

With (16) and (17), we can obtain an exact equality for the observation prediction error $\tilde{Z}_{k+1 \mid k}$.

$$
\tilde{\boldsymbol{Z}}_{k+1 \mid k}=\boldsymbol{B}_{k+1} \boldsymbol{H}_{k+1} \tilde{\boldsymbol{X}}_{k+1 \mid k}+\boldsymbol{v}_{k+1}
$$

where

$$
\begin{gathered}
\boldsymbol{H}_{k+1}=\left.[\partial \boldsymbol{h}(\boldsymbol{X}) / \partial \boldsymbol{X}]\right|_{\boldsymbol{X}=\hat{\boldsymbol{X}}_{k+1 \mid k}}, \\
\boldsymbol{B}_{k+1}=\operatorname{diag}\left\{b_{1, k+1}, \ldots, b_{n_{z}, k+1}\right\} .
\end{gathered}
$$

According to (14), the actual state prediction error covariance $\boldsymbol{P}_{k \mid k+1}$ can be obtained by

$$
\begin{aligned}
\boldsymbol{P}_{k+1 \mid k}= & \mathrm{E}\left[\left(\boldsymbol{A}_{k} \boldsymbol{F}_{k} \tilde{\boldsymbol{X}}_{k \mid k}+\boldsymbol{w}_{k}\right)\left(\boldsymbol{A}_{k} \boldsymbol{F}_{k} \tilde{\boldsymbol{X}}_{k \mid k}+\boldsymbol{w}_{k}\right)^{\mathrm{T}}\right]= \\
& \boldsymbol{A}_{k} \boldsymbol{F}_{k} \hat{\boldsymbol{P}}_{k \mid k} \boldsymbol{F}_{k}^{\mathrm{T}} \boldsymbol{A}_{k}+\phi \boldsymbol{P}_{k+1 \mid k}+\boldsymbol{Q}_{k}
\end{aligned}
$$

where $\phi \boldsymbol{P}_{k+1 \mid k}$ is the difference between $\mathrm{E}\left(\boldsymbol{A}_{k} \boldsymbol{F}_{k} \tilde{\boldsymbol{X}}_{k \mid k} \tilde{\boldsymbol{X}}_{k \mid k}^{\mathrm{T}} \boldsymbol{F}_{k}^{\mathrm{T}} \boldsymbol{A}_{k}\right)$ and $\boldsymbol{A}_{k} \boldsymbol{F}_{k} \hat{\boldsymbol{P}}_{k \mid k} \boldsymbol{F}_{k}^{\mathrm{T}} \boldsymbol{A}_{k}$.

Following [19], substituting (11) and (12) into (4) gives the prediction ECM $\hat{\boldsymbol{P}}_{k+1 \mid k}$ :

$$
\hat{\boldsymbol{P}}_{k+1 \mid k}=\boldsymbol{F}_{k} \hat{\boldsymbol{P}}_{k \mid k} \boldsymbol{F}_{k}^{\mathrm{T}}+\delta \hat{\boldsymbol{P}}_{k \mid k}+\boldsymbol{Q}_{k}
$$

where $\delta \hat{\boldsymbol{P}}_{k \mid k}$ is the combination of high-order terms.

Therefore, it can be obtained that

$$
\hat{\boldsymbol{P}}_{k+1 \mid k}=\boldsymbol{A}_{k} \boldsymbol{F}_{k} \hat{\boldsymbol{P}}_{k \mid k} \boldsymbol{F}_{k}^{\mathrm{T}} \boldsymbol{A}_{k}+\phi \boldsymbol{P}_{k+1 \mid k}+\varphi \boldsymbol{P}_{k+1 \mid k}+\boldsymbol{Q}_{k}=
$$

$$
\boldsymbol{A}_{k} \boldsymbol{F}_{k} \hat{\boldsymbol{P}}_{k \mid k} \boldsymbol{F}_{k}^{\mathrm{T}} \boldsymbol{A}_{k}+\hat{\boldsymbol{Q}}_{k}
$$

where

$$
\begin{gathered}
\hat{\boldsymbol{Q}}_{k}=\phi \boldsymbol{P}_{k+1 \mid k}+\varphi \boldsymbol{P}_{k+1 \mid k}+\boldsymbol{Q}_{k}, \\
\boldsymbol{\varphi} \boldsymbol{P}_{k+1 \mid k}=\hat{\boldsymbol{P}}_{k+1 \mid k}-\boldsymbol{P}_{k+1 \mid k} .
\end{gathered}
$$

Similarly, the following covariance can be expressed as

$$
\left\{\begin{array}{c}
\hat{\boldsymbol{P}}_{x z, k+1 \mid k}=\hat{\boldsymbol{P}}_{k+1 \mid k}\left(\boldsymbol{B}_{k+1} \boldsymbol{H}_{k+1}\right)^{\mathrm{T}}+ \\
\phi \boldsymbol{P}_{x z, k+1 \mid k}+\varphi \boldsymbol{P}_{x z, k+1 \mid k} \\
\hat{\boldsymbol{P}}_{z z, k+1 \mid k}=\boldsymbol{B}_{k+1} \boldsymbol{H}_{k+1} \hat{\boldsymbol{P}}_{k+1 \mid k}\left(\boldsymbol{B}_{k+1} \boldsymbol{H}_{k+1}\right)^{\mathrm{T}}+ \\
d_{k+1} \boldsymbol{R}_{k+1}+\left(1-d_{k+1}\right) \sigma^{2} \boldsymbol{I}+ \\
\phi \boldsymbol{P}_{z z, k+1 \mid k}+\varphi \boldsymbol{P}_{z z, k+1 \mid k}
\end{array}\right.
$$

Introducing the stochastic matrix $\boldsymbol{\mu}_{k+1} \in \mathbf{R}^{n_{x} \times n_{x}}$ [3], we have

$$
\hat{\boldsymbol{P}}_{x z, k+1 \mid k}=\hat{\boldsymbol{P}}_{k+1 \mid k} \boldsymbol{\mu}_{k+1}\left(\boldsymbol{B}_{k+1} \boldsymbol{H}_{k+1}\right)^{\mathrm{T}} .
$$

It can be obtained that

$$
\begin{gathered}
\hat{\boldsymbol{P}}_{k+1 \mid k+1}= \\
\hat{\boldsymbol{P}}_{k+1 \mid k}-\hat{\boldsymbol{P}}_{x z, k+1 \mid k} \lim _{\sigma^{2} \rightarrow \infty} \hat{\boldsymbol{P}}_{z z, k+1 \mid k}^{-1} \hat{\boldsymbol{P}}_{x z, k+1 \mid k}^{\mathrm{T}}= \\
\hat{\boldsymbol{P}}_{k+1 \mid k}-d_{k+1} \hat{\boldsymbol{P}}_{x z, k+1 \mid k} \cdot \\
{\left[\boldsymbol{B}_{k+1} \boldsymbol{H}_{k+1} \hat{\boldsymbol{P}}_{k+1 \mid k}\left(\boldsymbol{B}_{k+1} \boldsymbol{H}_{k+1}\right)^{\mathrm{T}}+\hat{\boldsymbol{R}}_{k+1}\right]^{-1} \hat{\boldsymbol{P}}_{x z, k+1 \mid k}^{\mathrm{T}}}
\end{gathered}
$$

where $\hat{\boldsymbol{R}}_{k+1}=\boldsymbol{R}_{k+1}+\phi \boldsymbol{P}_{z z, k+1 \mid k}+\varphi \boldsymbol{P}_{z z, k+1 \mid k}$.

Remark 1 Define $a_{k}^{\max }=\max _{j=1, \ldots, n_{x}}\left|a_{j, k}\right|$ and $b_{k}^{\max }=$ $\max _{j=1, \ldots, n_{z}}\left|b_{j, k}\right|$, as pointed out by [32], the upper bounds $j=1, \ldots, n_{z}$
$a_{k}^{\max }, b_{k}^{\max }$ can be estimated before the filtering is started. Generally, the estimated state $\hat{\boldsymbol{X}}_{k \mid k}$ will get close to the real state $\boldsymbol{X}_{k}$ as the growing of time step $k$, then the instrumental diagonal matrices $\boldsymbol{A}_{k}, \boldsymbol{B}_{k}$ will approach the identity matrices accordingly. In addition, it is worth mentioning that there is a major class of nonlinear systems with linear state equations, i.e., the radar target tracking system [21] with the state equation $\boldsymbol{X}_{k}=\boldsymbol{F}_{k} \boldsymbol{X}_{k-1}+\boldsymbol{w}_{k-1}$, in which we have $\boldsymbol{A}_{k}=\boldsymbol{I}_{n_{x}}$. Similarly, there also exist considerable nonlinear engineering systems with linear measurement equations, such as the two-phase nonlinear model of induction motor [18], where we have $\boldsymbol{Z}_{k}=$ $\boldsymbol{H}_{k} \boldsymbol{X}_{k}+\boldsymbol{v}_{k}$ and $\boldsymbol{B}_{k}=\boldsymbol{I}_{n_{z}}$. 


\section{Boundedness of the ECM of CKFI}

Before establishing the sufficient condition to ensure the boundedness of the ECM of CKFI, the following lemma and assumption are introduced.

Lemma 1 [27] For symmetric positive-definite matrices $\boldsymbol{C}, \boldsymbol{D} \in \mathbf{R}^{n \times n},(\boldsymbol{C}+\boldsymbol{D})^{-1}>\boldsymbol{C}^{-1}-\boldsymbol{C}^{-1} \boldsymbol{D} \boldsymbol{C}^{-1}$ holds.

Assumption 1 As pointed out in [28]: $\boldsymbol{H}_{k}^{-1}$ exists. There also exist real numbers $f_{\max }, h_{\min }, \mu_{\min }, \mu_{\max }$, $a_{\max }, b_{\min }, \hat{q}_{\max }, \hat{r}_{\max }>0$, such that

$$
\left\{\begin{array}{l}
\left\|\boldsymbol{F}_{k}\right\| \leqslant f_{\max } \\
h_{\min } \leqslant\left\|\boldsymbol{H}_{k}\right\| \\
\mu_{\min } \leqslant\left\|\boldsymbol{\mu}_{k}\right\| \leqslant \mu_{\max } \\
\boldsymbol{A}_{k} \leqslant a_{\max } \boldsymbol{I} \\
b_{\min } \boldsymbol{I} \leqslant \boldsymbol{B}_{k} \\
\hat{\boldsymbol{Q}}_{k} \leqslant \hat{q}_{\max } \boldsymbol{I} \\
\hat{\boldsymbol{R}}_{k} \leqslant \hat{r}_{\max } \boldsymbol{I}
\end{array} .\right.
$$

Theorem 1 If Assumption 1 is satisfied and the linear form of the nonlinear system in (1) is uniformly observable as in [27], there exists a critical value $\lambda_{c}=\frac{a_{\max }^{2} f_{\max }^{2}-1}{a_{\max }^{2} f_{\max }^{2} \mu_{\min }^{2}}$ such that the ECMs are bounded for the observation arrival probability $\lambda>\lambda_{c}$. That is, there exists a positive real constant $p_{\max }$ such that

$$
\mathrm{E}\left(\hat{\boldsymbol{P}}_{k+1 \mid k+1}\right) \leqslant \mathrm{E}\left(\hat{\boldsymbol{P}}_{k+1 \mid k}\right) \leqslant p_{\max } \boldsymbol{I} .
$$

Proof Substituting (24) into (21) gives

$$
\hat{\boldsymbol{P}}_{k+1 \mid k}=\boldsymbol{A}_{k} \boldsymbol{F}_{k}\left\{\hat{\boldsymbol{P}}_{k \mid k-1}-d_{k} \hat{\boldsymbol{P}}_{k \mid k-1} \boldsymbol{\mu}_{k}\left(\boldsymbol{B}_{k} \boldsymbol{H}_{k}\right)^{\mathrm{T}} .\right.
$$

$\left.\left[\boldsymbol{B}_{k} \boldsymbol{H}_{k} \hat{\boldsymbol{P}}_{k \mid k-1}\left(\boldsymbol{B}_{k} \boldsymbol{H}_{k}\right)^{\mathrm{T}}+\hat{\boldsymbol{R}}_{k}\right]^{-1}\left(\hat{\boldsymbol{P}}_{k \mid k-1} \boldsymbol{\mu}_{k} \boldsymbol{H}_{k}^{\mathrm{T}} \boldsymbol{B}_{k}\right)^{\mathrm{T}}\right\}$.

$$
\left(\boldsymbol{A}_{k} \boldsymbol{F}_{k}\right)^{\mathrm{T}}+\hat{\boldsymbol{Q}}_{k} .
$$

Setting $\boldsymbol{C}=\boldsymbol{B}_{k} \boldsymbol{H}_{k} \hat{\boldsymbol{P}}_{k \mid k-1}\left(\boldsymbol{B}_{k} \boldsymbol{H}_{k}\right)^{\mathrm{T}}, \boldsymbol{D}=\hat{\boldsymbol{R}}_{k}$ and applying Lemma 1 to the inverse term above yield

$$
\begin{gathered}
\hat{\boldsymbol{P}}_{k+1 \mid k} \leqslant \boldsymbol{A}_{k} \boldsymbol{F}_{k} \hat{\boldsymbol{P}}_{k \mid k-1}\left(\boldsymbol{A}_{k} \boldsymbol{F}_{k}\right)^{\mathrm{T}}- \\
d_{k} \boldsymbol{A}_{k} \boldsymbol{F}_{k} \hat{\boldsymbol{P}}_{k \mid k-1} \boldsymbol{\mu}_{k} \hat{\boldsymbol{P}}_{k \mid k-1}^{-1} \boldsymbol{\mu}_{k}^{\mathrm{T}} \hat{\boldsymbol{P}}_{k \mid k-1}^{\mathrm{T}}\left(\boldsymbol{A}_{k} \boldsymbol{F}_{k}\right)^{\mathrm{T}}+ \\
d_{k} \boldsymbol{A}_{k} \boldsymbol{F}_{k} \hat{\boldsymbol{P}}_{k \mid k-1} \boldsymbol{\mu}_{k} \hat{\boldsymbol{P}}_{k \mid k-1}^{-1}\left(\boldsymbol{B}_{k} \boldsymbol{H}_{k}\right)^{-1} \hat{\boldsymbol{R}}_{k}\left(\boldsymbol{B}_{k} \boldsymbol{H}_{k}\right)^{-\mathrm{T}} . \\
\hat{\boldsymbol{P}}_{k \mid k-1}^{-1} \boldsymbol{\mu}_{k}^{\mathrm{T}} \hat{\boldsymbol{P}}_{k \mid k-1}^{\mathrm{T}}\left(\boldsymbol{A}_{k} \boldsymbol{F}_{k}\right)^{\mathrm{T}}+\hat{\boldsymbol{Q}}_{k} .
\end{gathered}
$$

Considering Assumption 1, it can be derived that

$$
\begin{gathered}
\hat{\boldsymbol{P}}_{k+1 \mid k} \leqslant a_{\max }^{2} f_{\max }^{2}\left(1-d_{k} \mu_{\min }^{2}\right) \hat{\boldsymbol{P}}_{k \mid k-1}+ \\
\frac{d_{k} \hat{r}_{\max } a_{\max }^{2} f_{\max }^{2} \mu_{\max }^{2}+\hat{q}_{\max } h_{\min }^{2} b_{\min }^{2}}{h_{\min }^{2} b_{\min }^{2}} \boldsymbol{I} .
\end{gathered}
$$

Taking the mean value from both sides of (29) gives

$$
\mathrm{E}\left(\hat{\boldsymbol{P}}_{k+1 \mid k}\right) \leqslant a_{\max }^{2} f_{\max }^{2}\left(1-\lambda \mu_{\min }^{2}\right) \mathrm{E}\left(\hat{\boldsymbol{P}}_{k \mid k-1}\right)+
$$

$$
\frac{\lambda \hat{r}_{\max } a_{\max }^{2} f_{\max }^{2} \mu_{\max }^{2}+\hat{q}_{\max } h_{\min }^{2} b_{\min }^{2}}{h_{\min }^{2} b_{\min }^{2}} \boldsymbol{I} .
$$

Recursively, we reach (31) below.

$$
\begin{gathered}
\mathrm{E}\left(\hat{\boldsymbol{P}}_{k+1 \mid k}\right) \leqslant\left[a_{\max }^{2} f_{\max }^{2}\left(1-\lambda \mu_{\min }^{2}\right)\right]^{k}\left\|\hat{\boldsymbol{P}}_{1 \mid 0}\right\| \boldsymbol{I}+ \\
\frac{\lambda \cdot \hat{r}_{\max } a_{\max }^{2} f_{\max }^{2} \mu_{\max }^{2}+\hat{q}_{\max } h_{\min }^{2} b_{\min }^{2}}{h_{\min }^{2} b_{\min }^{2}} . \\
\sum_{i=0}^{k-1}\left[a_{\max }^{2} f_{\max }^{2}\left(1-\lambda \mu_{\min }^{2}\right)\right]^{i} \boldsymbol{I}
\end{gathered}
$$

Denote

$\hat{p}=\max \left\{\left\|\hat{\boldsymbol{P}}_{1 \mid 0}\right\|, \frac{\lambda \hat{r}_{\max } a_{\max }^{2} f_{\max }^{2} \mu_{\max }^{2}+\hat{q}_{\max } h_{\min }^{2} b_{\min }^{2}}{h_{\min }^{2} b_{\min }^{2}}\right\}$

then we have

$$
\mathrm{E}\left(\hat{\boldsymbol{P}}_{k+1 \mid k}\right) \leqslant \hat{p} \sum_{i=0}^{k}\left[a_{\max }^{2} f_{\max }^{2}\left(1-\lambda \mu_{\min }^{2}\right)\right]^{i} \boldsymbol{I} .
$$

It is obvious that under the assumption

$$
\lambda>\frac{a_{\max }^{2} f_{\max }^{2}-1}{a_{\max }^{2} f_{\max }^{2} \mu_{\min }^{2}},
$$

the sum in (32) converges to

$$
p_{\max }=\frac{\hat{p}}{1-a_{\max }^{2} f_{\max }^{2}\left(1-\lambda \mu_{\min }^{2}\right)} .
$$

Since $\mathrm{E}\left(\hat{\boldsymbol{P}}_{k+1 \mid k+1}\right) \leqslant \mathrm{E}\left(\hat{\boldsymbol{P}}_{k+1 \mid k}\right)$, the proof is completed.

Remark 2 Generally, the ECM for the nonlinear tracking filter is not in accordance with the state estimation error [28]. That is, the boundedness of ECM cannot necessarily guarantee the convergence of the estimation error. Therefore, the stochastic behavior of the estimation error $\tilde{\boldsymbol{X}}_{k \mid k}$ should be further studied.

\section{Stochastic stability of the estimation error of CKFI}

In this section, the estimation error results from the proposed CKFI will be shown to be bounded, if the following assumption and condition hold.

Lemma 2 [19] Assume that $\xi_{k}$ is the stochastic process, and there is a stochastic process $V_{k}\left(\xi_{k}\right)$ satisfying the following conditions for every $k \in N$ :

$$
\left\{\begin{array}{l}
v_{\min }\left\|\xi_{k}\right\|^{2} \leqslant V_{k}\left(\xi_{k}\right) \leqslant v_{\max }\left\|\xi_{k}\right\|^{2} \\
\mathrm{E}\left\{V_{k}\left(\xi_{k}\right) \mid \xi_{k-1}\right\}-V_{k-1}\left(\xi_{k-1}\right) \leqslant \rho-\tau V_{k-1}\left(\xi_{k-1}\right)
\end{array}\right.
$$


where $v_{\min }, v_{\max }, \rho>0$ and $0<\tau \leqslant 1$. Then the stochastic process $\xi_{k}$ is bounded in the mean square, and

$\mathrm{E}\left\{\left\|\xi_{k}\right\|^{2}\right\} \leqslant \frac{v_{\max }}{v_{\min }} \mathrm{E}\left\{\left\|\xi_{0}\right\|^{2}\right\}(1-\tau)^{k}+\frac{\rho}{v_{\min }} \sum_{i=1}^{k-1}(1-\tau)^{i}$.

Assumption 2 There exist real constants $f_{\max }, h_{\max }$, $\mu_{\text {max }}, a_{\text {max }}, b_{\text {max }}, \widehat{q}_{\text {min }}, \hat{r}_{\text {min }}, p_{\text {min }}, p_{\text {max }}>0$, such that the following bounds are fulfilled for every $k \in N$ :

$$
\left\{\begin{array}{l}
\left\|d_{k} \boldsymbol{F}_{k}\right\| \leqslant f_{\max } \\
\left\|d_{k} \boldsymbol{H}_{k}\right\| \leqslant h_{\max } \\
\left\|\boldsymbol{\mu}_{k}\right\| \leqslant \mu_{\max } \\
\boldsymbol{A}_{k} \leqslant a_{\max } \boldsymbol{I} \\
\boldsymbol{B}_{k} \leqslant b_{\max } \boldsymbol{I} \\
\widehat{q}_{\min } \boldsymbol{I} \leqslant \widehat{\boldsymbol{Q}}_{k} \\
\hat{r}_{\min } \boldsymbol{I} \leqslant \hat{\boldsymbol{R}}_{k} \\
p_{\min } \boldsymbol{I} \leqslant \hat{\boldsymbol{P}}_{k+1 \mid k} \leqslant p_{\max } \boldsymbol{I}
\end{array}\right.
$$

Theorem 2 Under Assumption 2, consider the nonlinear stochastic system described by (1) and the CKFI given by (3)-(8). If the initial estimation error satisfies $\mathrm{E}\left\{\left\|\tilde{\boldsymbol{X}}_{1 \mid 0}\right\|^{2}\right\} \leqslant \varepsilon$ for small real constant $\varepsilon>0$, and there exists a bound $\delta>0$ for the stochastic noise such that $\left\|\boldsymbol{w}_{k}^{\mathrm{T}} \boldsymbol{w}_{k}\right\| \leqslant \delta,\left\|\boldsymbol{v}_{k}^{\mathrm{T}} \boldsymbol{v}_{k}\right\| \leqslant \delta$. Then the estimation error $\tilde{\boldsymbol{X}}_{k \mid k}$ is exponentially bounded in the mean square and bounded with probability one.

Proof According to (8), (14) and (18), it can be obtained that

$$
\begin{gathered}
\tilde{\boldsymbol{X}}_{k+1 \mid k}=\boldsymbol{A}_{k} \boldsymbol{F}_{k}\left(\boldsymbol{I}-d_{k} \boldsymbol{K}_{k} \boldsymbol{B}_{k} \boldsymbol{H}_{k}\right) \tilde{\boldsymbol{X}}_{k \mid k-1}+\boldsymbol{s}_{k} \\
\boldsymbol{K}_{k}=\hat{\boldsymbol{P}}_{k \mid k-1} \boldsymbol{\mu}_{k}\left(\boldsymbol{B}_{k} \boldsymbol{H}_{k}\right)^{\mathrm{T}} \\
{\left[\boldsymbol{B}_{k} \boldsymbol{H}_{k} \hat{\boldsymbol{P}}_{k \mid k-1}\left(\boldsymbol{B}_{k} \boldsymbol{H}_{k}\right)^{\mathrm{T}}+\hat{\boldsymbol{R}}_{k}\right]^{-1}}
\end{gathered}
$$

where we denote

$$
\boldsymbol{s}_{k}=\boldsymbol{w}_{k}-d_{k} \boldsymbol{A}_{k} \boldsymbol{F}_{k} \boldsymbol{K}_{k} \boldsymbol{v}_{k}
$$

Define $V_{k+1}\left(\tilde{\boldsymbol{X}}_{k+1 \mid k}\right)=\tilde{\boldsymbol{X}}_{k+1 \mid k}^{\mathrm{T}} \hat{\boldsymbol{P}}_{k+1 \mid k}^{-1} \tilde{\boldsymbol{X}}_{k+1 \mid k}$, then

$$
\begin{gathered}
V_{k+1}\left(\tilde{\boldsymbol{X}}_{k+1 \mid k}\right)=\tilde{\boldsymbol{X}}_{k+1 \mid k}^{\mathrm{T}} \hat{\boldsymbol{P}}_{k+1 \mid k}^{-1} \tilde{\boldsymbol{X}}_{k+1 \mid k}= \\
\tilde{\boldsymbol{X}}_{k \mid k-1}^{\mathrm{T}}\left(\boldsymbol{I}-d_{k} \boldsymbol{K}_{k} \boldsymbol{B}_{k} \boldsymbol{H}_{k}\right)^{\mathrm{T}}\left(\boldsymbol{A}_{k} \boldsymbol{F}_{k}\right)^{\mathrm{T}} \\
\hat{\boldsymbol{P}}_{k+1 \mid k}^{-1} \boldsymbol{A}_{k} \boldsymbol{F}_{k}\left(\boldsymbol{I}-d_{k} \boldsymbol{K}_{k} \boldsymbol{B}_{k} \boldsymbol{H}_{k}\right) \tilde{\boldsymbol{X}}_{k \mid k-1}+ \\
2 \boldsymbol{s}_{k}^{\mathrm{T}} \hat{\boldsymbol{P}}_{k+1 \mid k}^{-1} \boldsymbol{A}_{k} \boldsymbol{F}_{k}\left(\boldsymbol{I}-d_{k} \boldsymbol{K}_{k} \boldsymbol{B}_{k} \boldsymbol{H}_{k}\right) \tilde{\boldsymbol{X}}_{k \mid k-1}+ \\
\boldsymbol{s}_{k}^{\mathrm{T}} \hat{\boldsymbol{P}}_{k+1 \mid k}^{-1} \boldsymbol{s}_{k} .
\end{gathered}
$$

Therefore, taking conditional expectation yields

$$
\mathrm{E}\left\{V_{k+1}\left(\tilde{\boldsymbol{X}}_{k+1 \mid k} \mid \tilde{\boldsymbol{X}}_{k \mid k-1}\right)\right\}=
$$

$$
\begin{gathered}
\mathrm{E}\left\{\tilde{\boldsymbol{X}}_{k \mid k-1}^{\mathrm{T}}\left(\boldsymbol{I}-d_{k} \boldsymbol{K}_{k} \boldsymbol{B}_{k} \boldsymbol{H}_{k}\right)^{\mathrm{T}}\left(\boldsymbol{A}_{k} \boldsymbol{F}_{k}\right)^{\mathrm{T}} .\right. \\
\left.\hat{\boldsymbol{P}}_{k+1 \mid k}^{-1} \boldsymbol{A}_{k} \boldsymbol{F}_{k}\left(\boldsymbol{I}-d_{k} \boldsymbol{K}_{k} \boldsymbol{B}_{k} \boldsymbol{H}_{k}\right) \tilde{\boldsymbol{X}}_{k \mid k-1}\right\}+ \\
\mathrm{E}\left\{\boldsymbol{s}_{k}^{\mathrm{T}} \hat{\boldsymbol{P}}_{k+1 \mid k}^{-1} \boldsymbol{s}_{k}\right\} .
\end{gathered}
$$

At the next step we take care of the first term above

$$
\begin{gathered}
\mathrm{E}\left\{\tilde{\boldsymbol{X}}_{k \mid k-1}^{\mathrm{T}}\left(\boldsymbol{I}-d_{k} \boldsymbol{K}_{k} \boldsymbol{B}_{k} \boldsymbol{H}_{k}\right)^{\mathrm{T}}\left(\boldsymbol{A}_{k} \boldsymbol{F}_{k}\right)^{\mathrm{T}} .\right. \\
\left.\hat{\boldsymbol{P}}_{k+1 \mid k}^{-1} \boldsymbol{A}_{k} \boldsymbol{F}_{k}\left(\boldsymbol{I}-d_{k} \boldsymbol{K}_{k} \boldsymbol{B}_{k} \boldsymbol{H}_{k}\right) \tilde{\boldsymbol{X}}_{k \mid k-1}\right\} .
\end{gathered}
$$

Based on (36), it follows

$$
\begin{gathered}
\hat{\boldsymbol{P}}_{k+1 \mid k}=\boldsymbol{A}_{k} \boldsymbol{F}_{k}\left(\boldsymbol{I}-d_{k} \boldsymbol{K}_{k} \boldsymbol{B}_{k} \boldsymbol{H}_{k}\right)\left\{\hat{\boldsymbol{P}}_{k \mid k-1}+\right. \\
{\left[\boldsymbol{A}_{k} \boldsymbol{F}_{k}\left(\boldsymbol{I}-d_{k} \boldsymbol{K}_{k} \boldsymbol{B}_{k} \boldsymbol{H}_{k}\right)\right]^{-1} \widehat{\boldsymbol{Q}}_{k} \cdot} \\
\left.\left[\boldsymbol{A}_{k} \boldsymbol{F}_{k}\left(\boldsymbol{I}-d_{k} \boldsymbol{K}_{k} \boldsymbol{B}_{k} \boldsymbol{H}_{k}\right)\right]^{-\mathrm{T}}\right\} \cdot \\
{\left[\boldsymbol{A}_{k} \boldsymbol{F}_{k}\left(\boldsymbol{I}-d_{k} \boldsymbol{K}_{k} \boldsymbol{B}_{k} \boldsymbol{H}_{k}\right)\right]^{\mathrm{T}}}
\end{gathered}
$$

where $\widehat{\boldsymbol{Q}}_{k}=\boldsymbol{s}_{k} \boldsymbol{s}_{k}^{\mathrm{T}}+\phi \boldsymbol{P}_{k+1 \mid k}+\varphi \boldsymbol{P}_{k+1 \mid k}$.

From (35) and (37), we have

$$
\left\|\boldsymbol{K}_{k}\right\| \leqslant \frac{p_{\max } \mu_{\max } h_{\max } b_{\max }}{\hat{r}_{\min }} \triangleq K_{\max } .
$$

On the basis of the characteristic of the matrix norm, it can be verified that

$$
\begin{gathered}
{\left[\boldsymbol{A}_{k} \boldsymbol{F}_{k}\left(\boldsymbol{I}-d_{k} \boldsymbol{K}_{k} \boldsymbol{B}_{k} \boldsymbol{H}_{k}\right)\right]^{-1} \widehat{\boldsymbol{Q}}_{k} .} \\
{\left[\boldsymbol{A}_{k} \boldsymbol{F}_{k}\left(\boldsymbol{I}-d_{k} \boldsymbol{K}_{k} \boldsymbol{B}_{k} \boldsymbol{H}_{k}\right)\right]^{-\mathrm{T}} \geqslant} \\
\frac{\widehat{q}_{\min } \boldsymbol{I}}{\left(a_{\max } f_{\max }+a_{\max } f_{\max } K_{\max } b_{\max } h_{\max }\right)^{2}} .
\end{gathered}
$$

According to (42), we establish that

$$
\hat{\boldsymbol{P}}_{k+1 \mid k} \geqslant\left[\boldsymbol{A}_{k} \boldsymbol{F}_{k}\left(\boldsymbol{I}-d_{k} \boldsymbol{K}_{k} \boldsymbol{B}_{k} \boldsymbol{H}_{k}\right)\right]
$$

$$
\begin{gathered}
{\left[\hat{\boldsymbol{P}}_{k \mid k-1}+\frac{\widehat{q}_{\min } \boldsymbol{I}}{\left(a_{\max } f_{\max }+a_{\max } f_{\max } K_{\max } b_{\max } h_{\max }\right)^{2}}\right]} \\
{\left[\boldsymbol{A}_{k} \boldsymbol{F}_{k}\left(\boldsymbol{I}-d_{k} \boldsymbol{K}_{k} \boldsymbol{B}_{k} \boldsymbol{H}_{k}\right)\right]^{\mathrm{T}}}
\end{gathered}
$$

Taking the inverse of both sides of (45), it can be further derived that

$$
\begin{gathered}
\hat{\boldsymbol{P}}_{k+1 \mid k}^{-1} \leqslant\left[\boldsymbol{A}_{k} \boldsymbol{F}_{k}\left(\boldsymbol{I}-d_{k} \boldsymbol{K}_{k} \boldsymbol{B}_{k} \boldsymbol{H}_{k}\right)\right]^{-\mathrm{T}} . \\
{\left[\hat{\boldsymbol{P}}_{k \mid k-1}+\frac{\widehat{q}_{\min } \boldsymbol{I}}{\left(a_{\max } f_{\max }+a_{\max } f_{\max } K_{\max } b_{\max } h_{\max }\right)^{2}}\right]^{-1} .} \\
{\left[\boldsymbol{A}_{k} \boldsymbol{F}_{k}\left(\boldsymbol{I}-d_{k} \boldsymbol{K}_{k} \boldsymbol{B}_{k} \boldsymbol{H}_{k}\right)\right]^{-1} .}
\end{gathered}
$$

Using (35) we can obtain (47) below.

$$
\left[\boldsymbol{A}_{k} \boldsymbol{F}_{k}\left(\boldsymbol{I}-d_{k} \boldsymbol{K}_{k} \boldsymbol{B}_{k} \boldsymbol{H}_{k}\right)\right]^{\mathrm{T}} \hat{\boldsymbol{P}}_{k+1 \mid k}^{-1} .
$$




$$
\begin{gathered}
{\left[\boldsymbol{A}_{k} \boldsymbol{F}_{k}\left(\boldsymbol{I}-d_{k} \boldsymbol{K}_{k} \boldsymbol{B}_{k} \boldsymbol{H}_{k}\right)\right] \leqslant} \\
{\left[\boldsymbol{I}+\frac{\widehat{q}_{\min } \boldsymbol{I}}{p_{\max }\left(a_{\max } f_{\max }+a_{\max } f_{\max } K_{\max } b_{\max } h_{\max }\right)^{2}}\right]^{-1}} \\
\hat{\boldsymbol{P}}_{k \mid k-1}^{-1} .
\end{gathered}
$$

Define

$$
1-\alpha \triangleq
$$

$\frac{p_{\max }\left(a_{\max } f_{\max }+a_{\max } f_{\max } K_{\max } b_{\max } h_{\max }\right)^{2}}{p_{\max }\left(a_{\max } f_{\max }+a_{\max } f_{\max } K_{\max } b_{\max } h_{\max }\right)^{2}+\widehat{q}_{\min }}$,

$0<\alpha<1$, the first term of (40) is bounded.

The second term of (40) is $\mathrm{E}\left\{\boldsymbol{s}_{k}^{\mathrm{T}} \hat{\boldsymbol{P}}_{k+1 \mid k}^{-1} \boldsymbol{s}_{k}\right\}$, substituting (38) into it yields

$$
\begin{gathered}
\mathrm{E}\left\{\boldsymbol{s}_{k}^{\mathrm{T}} \hat{\boldsymbol{P}}_{k+1 \mid k}^{-1} \boldsymbol{s}_{k}\right\}=\mathrm{E}\left\{\boldsymbol{w}_{k}^{\mathrm{T}} \hat{\boldsymbol{P}}_{k+1 \mid k}^{-1} \boldsymbol{w}_{k}\right\}+ \\
\mathrm{E}\left\{\boldsymbol{v}_{k}^{\mathrm{T}}\left[\boldsymbol{A}_{k} d_{k} \boldsymbol{F}_{k} \boldsymbol{K}_{k}\right]^{\mathrm{T}} \hat{\boldsymbol{P}}_{k+1 \mid k}^{-1}\left[\boldsymbol{A}_{k} d_{k} \boldsymbol{F}_{k} \boldsymbol{K}_{k}\right] \boldsymbol{v}_{k}\right\} \leqslant \\
\frac{\left\|\boldsymbol{w}_{k}^{\mathrm{T}} \boldsymbol{w}_{k}\right\|+a_{\max }^{2} f_{\max }^{2} K_{\max }^{2}\left\|\boldsymbol{v}_{k}^{\mathrm{T}} \boldsymbol{v}_{k}\right\|}{p_{\min }} .
\end{gathered}
$$

Considering that $\left\|\boldsymbol{w}_{k}^{\mathrm{T}} \boldsymbol{w}_{k}\right\| \leqslant \delta,\left\|\boldsymbol{v}_{k}^{\mathrm{T}} \boldsymbol{v}_{k}\right\| \leqslant \delta$, it can be readily obtained that

$$
\mathrm{E}\left\{\boldsymbol{s}_{k}^{\mathrm{T}} \hat{\boldsymbol{P}}_{k+1 \mid k}^{-1} \boldsymbol{s}_{k}\right\} \leqslant \frac{\delta+a_{\max }^{2} f_{\max }^{2} K_{\max }^{2} \delta}{p_{\min }} .
$$

Denote $\kappa_{s}=\frac{\delta+a_{\max }^{2} f_{\max }^{2} K_{\max }^{2} \delta}{p_{\min }}$, combining (47) and (49) and using (35) we have

$$
\begin{gathered}
\mathrm{E}\left\{V_{k+1}\left(\tilde{\boldsymbol{X}}_{k+1 \mid k} \mid \tilde{\boldsymbol{X}}_{k \mid k-1}\right)\right\}-V_{k}\left(\tilde{\boldsymbol{X}}_{k \mid k-1}\right) \leqslant \\
\kappa_{s}-\alpha V_{k}\left(\tilde{\boldsymbol{X}}_{k \mid k-1}\right), \\
\frac{\left\|\tilde{\boldsymbol{X}}_{k+1 \mid k}\right\|}{p_{\max }} \leqslant V_{k+1}\left(\tilde{\boldsymbol{X}}_{k+1 \mid k}\right) \leqslant \frac{\left\|\tilde{\boldsymbol{X}}_{k+1 \mid k}\right\|}{p_{\min }} .
\end{gathered}
$$

Thus, by applying Lemma 2 , the estimation error $\tilde{\boldsymbol{X}}_{k+1 \mid k}$ meets

$$
\begin{gathered}
\mathrm{E}\left\{\left\|\tilde{\boldsymbol{X}}_{k+1 \mid k}\right\|^{2}\right\} \leqslant \frac{p_{\max }}{p_{\min }} \mathrm{E}\left\{\left\|\tilde{\boldsymbol{X}}_{1 \mid 0}\right\|^{2}\right\}(1-\alpha)^{k}+ \\
p_{\max } \kappa_{s} \sum_{i=1}^{k-1}(1-\alpha)^{i} .
\end{gathered}
$$

By (14), it can be easily derived that $\mathrm{E}\left\{\left\|\tilde{\boldsymbol{X}}_{k \mid k}\right\|^{2}\right\} \leqslant$ $\frac{2\left\{\mathrm{E}\left\{\left\|\tilde{\boldsymbol{X}}_{k+1 \mid k}\right\|^{2}\right\}+\mathrm{E}\left\{\left\|\boldsymbol{w}_{k}\right\|^{2}\right\}\right\}}{\alpha_{\min }^{2} f_{\min }^{2}}$.

Hence, we can easily draw the conclusion that the estimation error $\tilde{\boldsymbol{X}}_{k \mid k}$ of the CKFI is bounded in mean square.

\section{Illustrative examples}

Example 1 The first nonlinear system that we consider can be described [30] as

$$
\left\{\begin{array}{l}
x_{k}=1.1 x_{k-1}+0.2 \sin \left(x_{k-1}\right)+w_{k-1} \\
z_{k}=d_{k} x_{k}+v_{k}
\end{array}\right.
$$

where $w_{k}, v_{k}$ are the zero-mean white Gaussian noises with covariance $Q_{k}=0.01$ and $R_{k}=6$, respectively. The initial conditions are $x_{0}=0.1, \hat{x}_{0 \mid 0}=0$, the initial state error covariance is $\hat{p}_{0 \mid 0}=1.0^{2}$.

From (52), we can derive that $F_{k}=\partial[f(x)] / \partial x_{k}=$ $1.1+0.2 \cos \left(x_{k}\right)$, thus it can be verified by numerical simulation that $f_{\max }=1.30, \mu_{\min }=0.92, a_{\max }=$ 0.83 . According to Theorem 1 , the sufficient condition for boundedness of the ECM is $\lambda>\left(a_{\max }^{2} f_{\max }^{2}-\right.$ 1) $/\left(a_{\max }^{2} f_{\max }^{2} \mu_{\text {min }}^{2}\right)=0.16$.

As can be seen from Fig. 1, for the nonlinear system given in (52), there exists a critical value $\lambda_{c}=$ 0.16 for the observation arrival probability such that the ECM is bounded, which verifies the theoretical result in Theorem 1 .

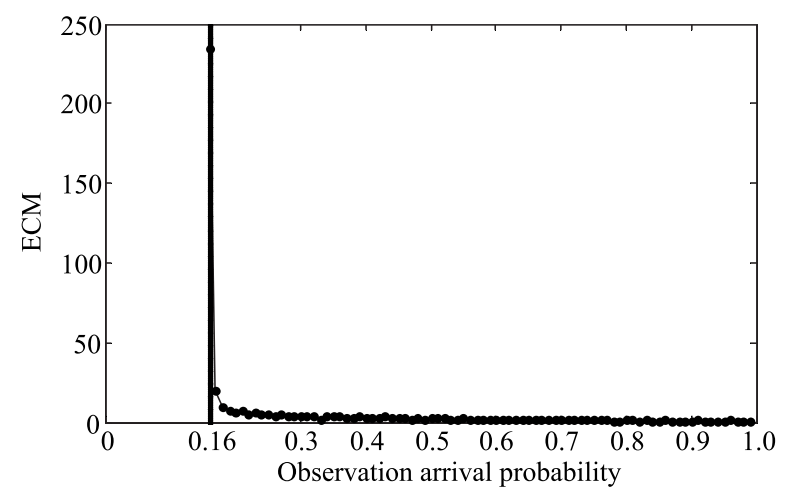

Fig. 1 ECM of the CKFI with different observation arrival probabilities

Example 2 The second numerical example concerns another nonlinear stochastic system [1,3], where $\tau=$ 0.006 , covariance matrices of the state noise $\boldsymbol{w}_{k}$ and measurement noise $v_{k}$ are $\boldsymbol{Q}_{k}=0.003^{2} \boldsymbol{I}, R_{k}=0.01^{2}$, respectively. The initial state is given as $\boldsymbol{X}_{0}=\left[\begin{array}{ll}0.8 & 0.2\end{array}\right]^{\mathrm{T}}$.

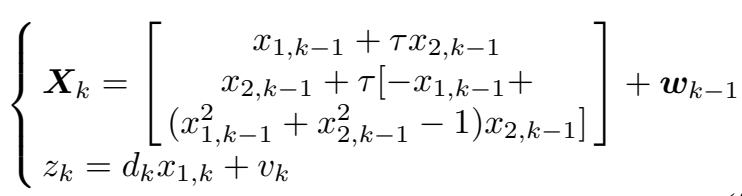

According to Theorems 1-2, the estimation error of the proposed CKFI will be bounded under some assumptions and appropriate initial conditions. Hence, in our simulation, three cases are taken into consideration: small initial 
error with a high observation arrival probability, small initial error but with a low observation arrival probability, and large initial error as well as a high observation arrival probability. The detailed parameters are demonstrated in Table 1.

Table 1 Initial error and arrival probability

\begin{tabular}{cccc}
\hline Item & $\begin{array}{c}\text { Small initial error } \\
\text { and high arrival } \\
\text { probability }\end{array}$ & $\begin{array}{c}\text { Low arrival } \\
\text { probability }\end{array}$ & $\begin{array}{c}\text { Large initial } \\
\text { error }\end{array}$ \\
\hline$\hat{\boldsymbol{X}}_{0}$ & {$\left[\begin{array}{ccc}0.9 & 0.3\end{array}\right]^{\mathrm{T}}$} & {$\left[\begin{array}{cc}0.9 & 0.3\end{array}\right]^{\mathrm{T}}$} & {$\left[\begin{array}{cc}5.8 & 5.2\end{array}\right]^{\mathrm{T}}$} \\
$\lambda$ & 0.8 & 0.1 & 0.8 \\
Figure & 2 & 3 & 4 \\
\hline
\end{tabular}

The simulation results are demonstrated in Figs. 2-4, where the true state $x_{2, k}$, the estimated state $\hat{x}_{2, k}$ and the estimation error $\tilde{x}_{2, k}$ are depicted for three conditions in Table 1. From Fig. 2, we can see that the estimation error keeps bounded for the small initial error and a high observation arrival probability. As shown in Fig. 3, the low observation arrival probability will result in the divergence of the ECM and lead to the unbounded estimation error, which is in accordance with Theorem 1. According to Theorem 2, the estimation error will also diverge due to the large initial error as demonstrated in Fig. 4.

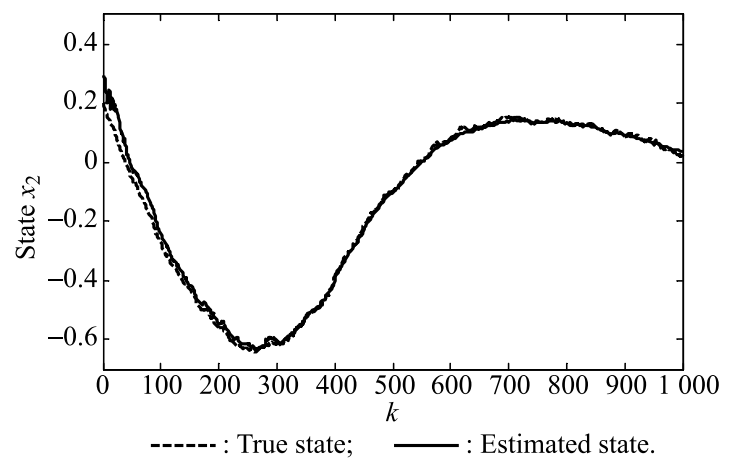

(a) Estimated state $\hat{x}_{2, k}$

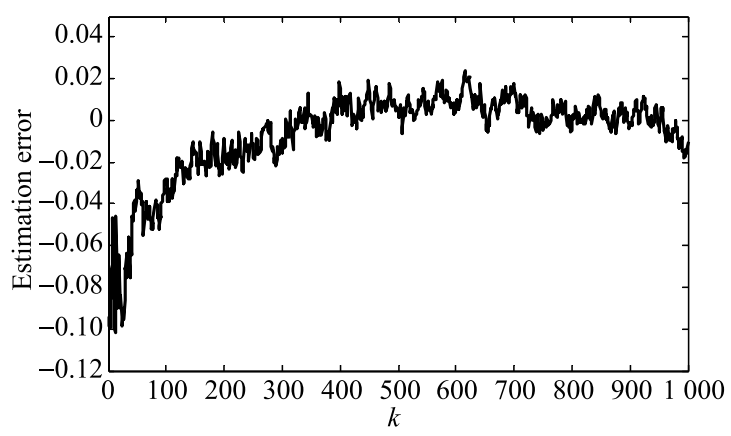

(b) Estimation error $\widetilde{x}_{2, k}$

Fig. 2 Simulation results with $\hat{X}_{0}=\left[\begin{array}{ll}0.9 & 0.3\end{array}\right]^{\mathrm{T}}$ and $\lambda=0.8$

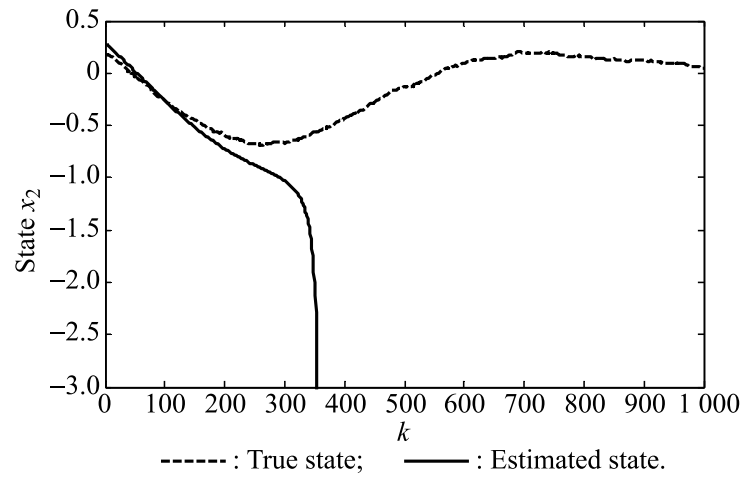

(a) Estimated state $\hat{x}_{2, k}$

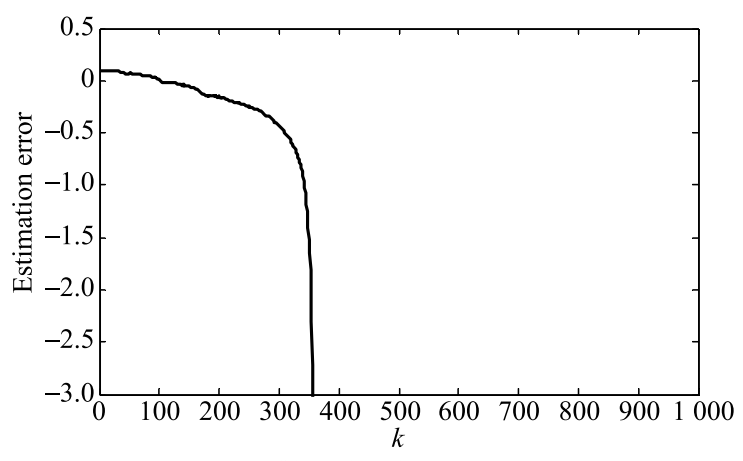

(b) Estimation error $\widetilde{x}_{2, k}$

Fig. 3 Simulation results with $\hat{X}_{0}=\left[\begin{array}{ll}0.9 & 0.3\end{array}\right]^{\mathrm{T}}$ and $\lambda=0.1$

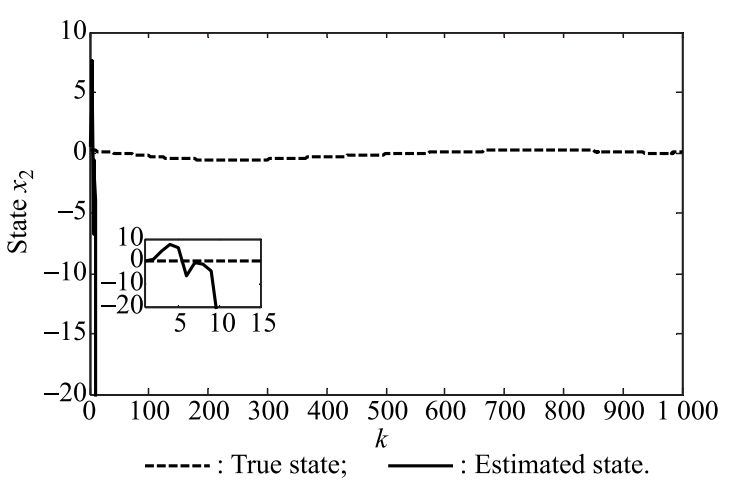

(a) Estimated state $\hat{x}_{2, k}$

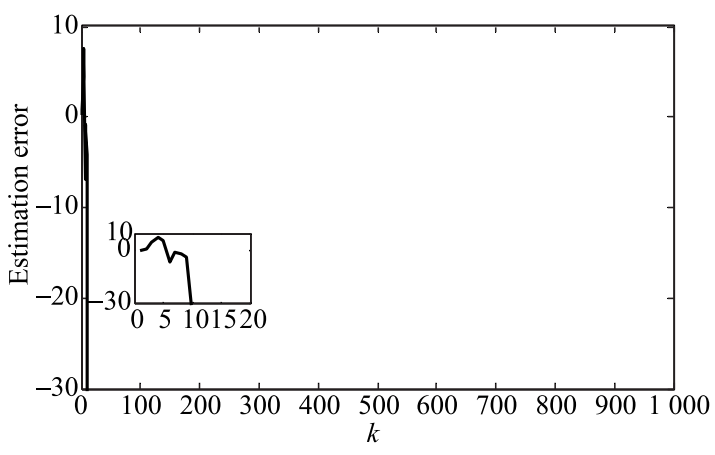

(b) Estimation error $\widetilde{x}_{2, k}$

Fig. 4 Simulation results with $\hat{X}_{0}=\left[\begin{array}{ll}5.8 & 5.2\end{array}\right]^{\mathrm{T}}$ and $\lambda=0.8$ 
Example 3 Application to the optic-electric tracking system

The previous two examples have been utilized to verify the theoretical results in this article. To evaluate the estimation performance of the filtering framework provided in Section 2, the proposed CKFI will be applied to the optic-electric tracking system in this example. As shown in Fig. 5, the azimuth angle $\beta_{k}$, elevation angle $\varepsilon_{k}$ are measured according to the visible/infrared image-based target tracking, also the laser rangefinder is adopted to obtain the target distance $D_{k}$. Combining with the practical application, the observations of the optic-electric tracking system may be lost due to the cloud cover, a high noise environment, intermittent sensor failures, accidental loss of some collected data, etc.

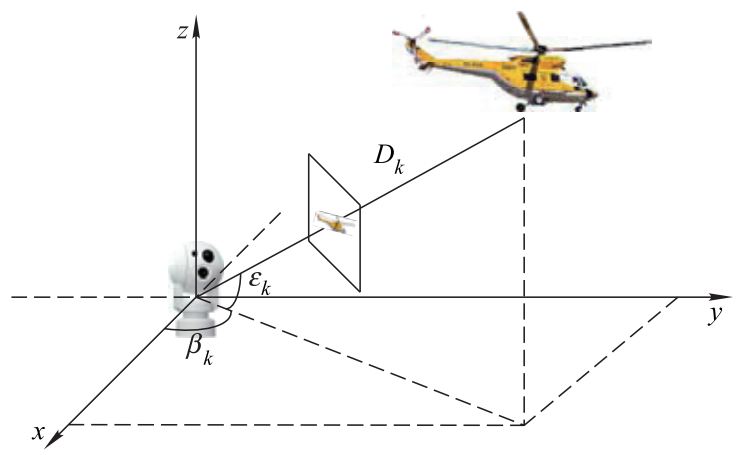

Fig. 5 Schematic diagram of the optic-electric tracking system

We define the target state as

$$
\boldsymbol{X}_{k}=\left[x_{k}, \dot{x}_{k}, y_{k}, \dot{y}_{k}, z_{k}, \dot{z}_{k}\right]^{\mathrm{T}}
$$

where $\left[x_{k}, y_{k}, z_{k}\right]^{\mathrm{T}}$ indicates the target position in the Cartesian coordinate, and $\left[\dot{x}_{k}, \dot{y}_{k}, \dot{z}_{k}\right]$ denotes the corresponding target velocity, the tracking equation of the opticelectric tracking system can be obtained by

$$
\left\{\begin{aligned}
& \boldsymbol{X}_{k}= \boldsymbol{F}_{k} \boldsymbol{X}_{k-1}+\boldsymbol{w}_{k-1} \\
& \boldsymbol{Z}_{k}=d_{k}\left[\begin{array}{l}
D_{k} \\
\beta_{k} \\
\varepsilon_{k}
\end{array}\right]+\boldsymbol{v}_{k}= \\
& d_{k}\left[\begin{array}{c}
\sqrt{x_{k}^{2}+y_{k}^{2}+z_{k}^{2}} \\
\arctan \left(y_{k} / x_{k}\right) \\
\arctan \left(z_{k} / \sqrt{x_{k}^{2}+y_{k}^{2}}\right)
\end{array}\right]+\left[\begin{array}{c}
v_{k}^{d} \\
v_{k}^{\mathcal{\beta}} \\
v_{k}^{\varepsilon}
\end{array}\right]
\end{aligned}\right.
$$

where $\boldsymbol{F}_{k} \in \mathbf{R}^{6 \times 6}$ is the state transitional matrix. $\boldsymbol{w}_{k} \in$ $\mathbf{R}^{6}$ is white Gaussian noise with zero mean and covariance matrix $\boldsymbol{Q}_{k} \in \mathbf{R}^{6 \times 6}$. $v_{k}^{d}, v_{k}^{\beta}$ and $v_{k}^{\varepsilon}$ are independent white Gaussian noises with covariance $\sigma_{d}^{2}, \sigma_{\beta}^{2}$ and $\sigma_{\varepsilon}^{2}$, respectively. $d_{k}$ is a scalar binary Bernoulli distributed random variable that indicates whether the observations are achieved. Without loss of generality, the constant velocity
(CV) target model is adopted in our example [21], and we have

$$
\left\{\begin{array}{l}
\boldsymbol{F}_{k}=\boldsymbol{I}_{3} \otimes\left[\begin{array}{cc}
1 & T \\
0 & 1
\end{array}\right] \\
\boldsymbol{Q}_{k}=\boldsymbol{I}_{3} \otimes\left[\begin{array}{cc}
T^{3} / 3 & T^{2} / 2 \\
T^{2} / 2 & T
\end{array}\right] q^{2}
\end{array}\right.
$$

where $\otimes$ is the Kronecker product, $T$ is the sample interval, and $q$ is the process noise intensity.

In our implementation, the sample interval $T=$ $0.2 \mathrm{~s}$, the process noise intensity $q=1 \mathrm{~m} / \mathrm{s}^{2}$. The optic-electric tracking system is static and at the origin of the coordinate system, while the target is at location $(4000 \mathrm{~m}, 4000 \mathrm{~m}, 1500 \mathrm{~m})$ with the velocity $(50 \mathrm{~m} / \mathrm{s},-50 \mathrm{~m} / \mathrm{s}, 0 \mathrm{~m} / \mathrm{s})$. The observation arrival probability $\lambda=0.8$ [21]. The measurement accuracy can be given as $\sigma_{d}=5 \mathrm{~m}, \sigma_{\beta}=\sigma_{\varepsilon}=5 \mathrm{mil},(2 \pi=6000 \mathrm{mil})$. Generally, the estimation performance of the tracking filter can be evaluated by the root mean square error (RMSE) and accumulative root mean square error (ARMSE), the Monte Carlo runs $M=100$.

The error comparisons are performed between the proposed CKFI and the UKFI. In Fig. 6, the estimated target trajectories by two filters are shown. Meantime, the RMSEs of CKFI and UKFI are depicted in Fig. 7.

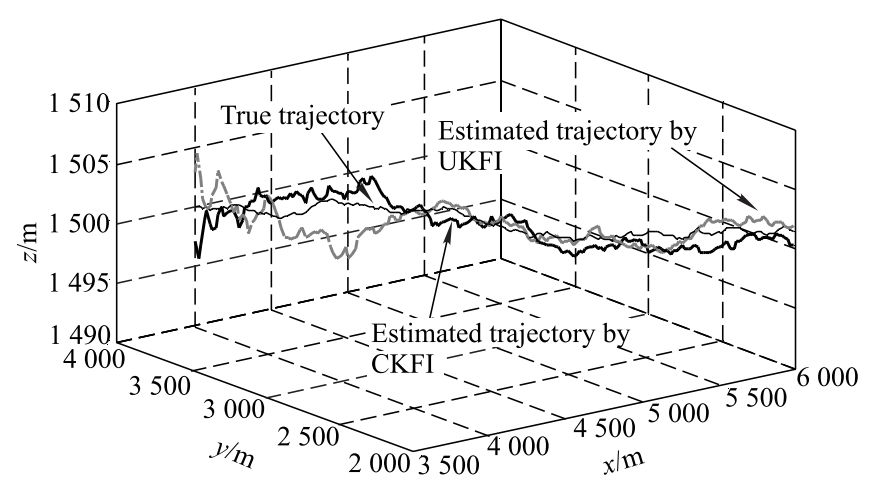

Fig. 6 The estimated trajectories by CKFI and UKFI

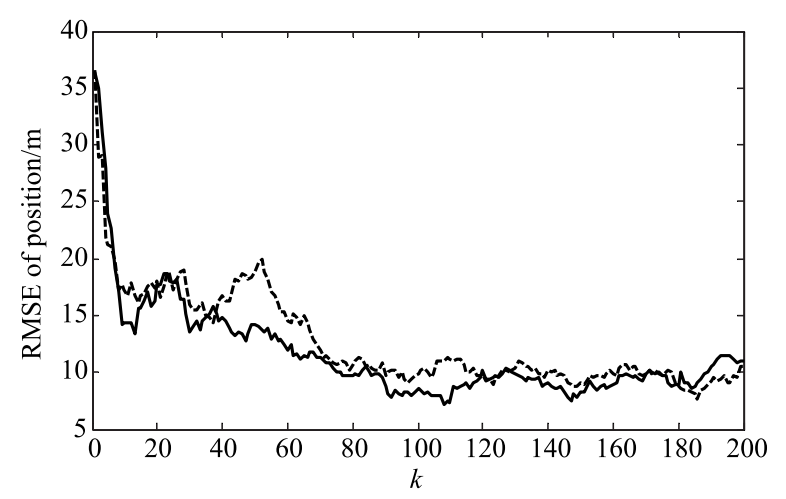

(a) RMSE of position 


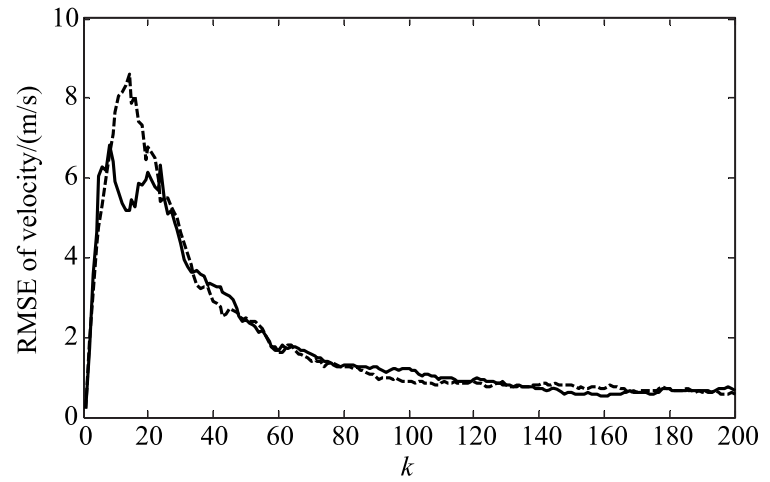

(b) RMSE of velocity

: CKFI; -----.. : UKFI

Fig. 7 Comparison of RMSEs with the proposed CKFI and UKFI

Furthermore, in Table 2, the ARMSEs of position and velocity, as well as the mean computational time per filtering running in Matlab 2014a on a $3.0 \mathrm{GHz}, 4 \mathrm{G}$ memorybased Windows workstation are presented.

Table 2 Performance metrics of the CKFI and UKFI

\begin{tabular}{cccc}
\hline Filter & $\begin{array}{c}\text { ARMSE of } \\
\text { position } / \mathrm{m}\end{array}$ & $\begin{array}{c}\text { ARMSE of } \\
\text { velocity } /(\mathrm{m} / \mathrm{s})\end{array}$ & $\begin{array}{c}\text { Mean computational } \\
\text { time/s }\end{array}$ \\
\hline CKFI & 11.55 & 1.85 & 8.82 \\
UKFI & 12.60 & 1.93 & 9.45 \\
\hline
\end{tabular}

It can be concluded from Fig. 7 and Table 2 as follows:

(i) The CKFI slighter outperforms the UKFI in terms of tracking accuracy, that is, both the RMSEs and ARMSEs of the CKFI are a bit smaller than that of the UKFI.

(ii) As compared to the UKFI, the CKFI is an effective method with shorter mean computational time, which is of significance in practical target tracking. The main reason for this conclusion is that the CKFI uses fewer sampling points than the UKFI.

In addition, to test the influence of the observation arrival probability on filtering performance, as shown in Table $3, \lambda$ are designed as $\lambda=0.6, \lambda=0.7$ and $\lambda=0.8$. From Table 3 , it is obvious that the estimation precision of CKFI is higher with the higher observation arrival probability. According to Fig. 7 and Tables $2-3$, it is clear that the CKFI is practical and effective; meantime, the estimation performance of the CKFI can be influenced by the observation arrival probability.

Table 3 Comparison of ARMSEs of the CKFI with different probabilities

\begin{tabular}{cccc}
\hline ARMSE & $\lambda=0.6$ & $\lambda=0.7$ & $\lambda=0.8$ \\
\hline Position $/ \mathrm{m}$ & 13.79 & 12.52 & 11.55 \\
Velocity $/(\mathrm{m} / \mathrm{s})$ & 2.03 & 1.95 & 1.85 \\
\hline
\end{tabular}

\section{Conclusions}

This study addresses the stochastic stability of CKF for discrete-time nonlinear systems with intermittent observations. The estimation error and ECM of CKFI are firstly obtained by Taylor expansion. Then, it can be proven that there exists a critical minimum observation arrival probability such that the ECM is bounded, and certain conditions for guaranteeing the stability of the estimation error are deduced. Finally, as compared to UKFI, the better performances of CKFI are demonstrated through the application to the optic-electric target tracking system.

Further research topic could be the extension of CKF for the stochastic nonlinear systems with Markovian observation dropouts. Moreover, it is of significance to study the CKF-based estimation methods under the condition of random observation delays.

\section{References}

[1] REIF K, GUNTHER S, YAZ E, et al. Stochastic stability of the discrete-time extended Kalman filter. IEEE Trans. on Automatic Control, 1999, 44(4): 714-728.

[2] JULIER S, UHLMANN J, DURRANT-WHYTE H F. A new method for the nonlinear transformation of means and covariances in filters and estimators. IEEE Trans. on Automatic Control, 2000, 45(3): $477-482$.

[3] XIONG K, ZHANG H Y, CHAN C W. Performance evaluation of UKF-based nonlinear filtering. Automatica, 2006, 42(2): $261-270$.

[4] ARULAMPALAM M S, MASKELL S, GORDON N, et al. A tutorial on particle filters for online nonlinear/non-Gaussian Bayesian tracking. IEEE Trans. on Signal Processing, 2002, 50(2): $174-188$

[5] SPEEKENBRINK M. A tutorial on particle filters. Journal of Mathematical Psychology, 2016, 73(8): 140-152.

[6] OH S, HAHN M, KIM J. Dynamic EKF-based SLAM for autonomous mobile convergence platforms. Multimedia Tools and Applications, 2015, 74(16): 6413-6430.

[7] SINGH A K, PAL B C. Decentralized dynamic state estimation in power systems using unscented transformation. IEEE Trans. on Power Systems, 2014, 29(2): 794-804.

[8] FOO P H. Combining the interacting multiple model method with particle filters for manoeuvring target tracking with a multistatic radar system. IET Radar, Sonar \& Navigation, 2011, 5(7): $697-706$

[9] ARASARATNAM I, HAYKIN S. Cubature Kalman filters. IEEE Trans. on Automatic Control, 2009, 54(6): 1254-1269.

[10] ARASARATNAM I, HAYKIN S, HURD T R. Cubature Kalman filtering for continuous-discrete systems: theory and simulations. IEEE Trans. on Signal Processing, 2010, 58(10): $4977-4993$.

[11] JIA B, XIN M, CHENG Y. High-degree cubature Kalman filter. Automatica, 2013, 49(2): 510-518.

[12] GE Q B, XU D X, WEN C L. Cubature information filters with correlated noises and their applications in decentralized fusion. Signal Processing, 2014, 94(1): 434-444.

[13] ZAREI J, SHOKRI E. Nonlinear and constrained state estimation based on the cubature Kalman filter. Industrial \& Engineering Chemistry Research, 2014, 53(10): 3938 - 3949.

[14] ZHANG L J, YANG H B, LU H P, et al. Cubature Kalman filtering for relative spacecraft attitude and position estimation. Acta Astronautica, 2014, 105(1): 254-264.

[15] ZHU W, WANG W, YUAN G N. An improved interact- 
ing multiple model filtering algorithm based on the cubature Kalman filter for maneuvering target tracking. Sensors, 2016, 16(6): 805.

[16] WU H, CHEN S X, YANG B F, et al. Robust derivative-free cubature Kalman filter for bearings-only tracking. Journal of Guidance, Control, and Dynamics, 2016, 39(8): 1866-1871.

[17] ZHAO Y W. Performance evaluation of cubature Kalman filter in a GPS/IMU tightly-coupled navigation system. Signal Processing, 2016, 119(C): 67-79.

[18] ZAREI J, SHOKRI E. Convergence analysis of non-linear filtering based on cubature Kalman filter. IET Science, Measurement \& Technology, 2014, 9(3): 294-305.

[19] XU B, ZHANG P, WEN H Z, et al. Stochastic stability and performance analysis of cubature Kalman filter. Neurocomputing, 2016, 186(4): $218-227$.

[20] DONG H L, WANG Z D, HO D W C, et al. Varianceconstrained $\mathrm{H}_{\infty}$ filtering for a class of nonlinear time-varying systems with multiple missing measurements: the finitehorizon case. IEEE Trans. on Signal Processing, 2010, 58(5): $2534-2543$.

[21] SHI J, LI Y Y, QI G Q, et al. Extended target tracking filter with intermittent observations. IET Signal Processing, 2016, 10(6): $592-602$.

[22] ZHANG W A, YU L, SONG H B. $\mathrm{H}_{\infty}$ filtering of networked discrete-time systems with random packet losses. Information Sciences, 2009, 179(22): 3944-3955.

[23] SUN S L, MA J. Linear estimation for networked control systems with random transmission delays and packet dropouts. Information Sciences, 2014, 269(4): 349-365.

[24] SINOPOLI B, SCHENATO L, FRANCESCHETTI M, et al. Kalman filtering with intermittent observations. IEEE Trans. on Automatic Control, 2004, 49(9): 1453-1464.

[25] HUANG M Y, DEY S. Stability of Kalman filtering with Markovian packet losses. Automatica, 2007, 43(4): 598-607.

[26] ROHR E R, MARELLI D, FU M. Kalman filtering with intermittent observations: on the boundedness of the expected error covariance. IEEE Trans. on Automatic Control, 2014, 59(10): $2724-2738$.

[27] KLUGE S, REIF K, BROKATE M. Stochastic stability of the extended Kalman filter with intermittent observations. IEEE Trans. on Automatic Control, 2010, 55(2): 514-518.

[28] WANG G, CHEN J, SUN J. Stochastic stability of extended filtering for non-linear systems with measurement packet losses. IET Control Theory \& Applications, 2013, 7(17): $2048-$ 2055.

[29] LI L, XIA Y Q. Stochastic stability of the unscented Kalman filter with intermittent observations. Automatica, 2012, 48(5): $978-981$.

[30] FOLETTO T D C, MORENO U F. On the performance of unscented Kalman filter with intermittent observations. Proc. of the 12th IEEE International Conference on Industrial Infor- matics, 2014: 660-665.

[31] LI L, XIA Y Q. Unscented Kalman filter over unreliable communication networks with Markovian packet dropouts. IEEE Trans. on Automatic Control, 2013, 58(12): 3224-3230.

[32] BOUTAYEB M, AUBRY D. A strong tracking extended Kalman observer for nonlinear discrete-time systems. IEEE Trans. on Automatic Control, 1999, 44(8): 1550-1556.

\section{Biographies}

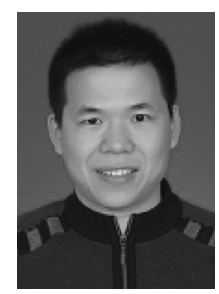

SHI Jie was born in 1988. He received his Ph.D. degree at the School of Automation, Nanjing University of Science and Technology in 2017. He is currently an engineer of NARI Group Corporation in Nanjing. His major research interest is nonlinear filtering.

E-mail: sjwyf6288@163.com

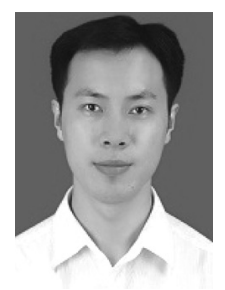

QI Guoqing was born in 1977. He received his B.S. degree and Ph.D. degree from Nanjing University of Science and Technology in 2002 and 2006 respectively. He has been an associate professor with School of Automation, Nanjing University of Science and Technology, since 2006. His major research interests are nonlinear filtering, fire control tracking and distributed estimation.

E-mail: qiguoqing@mail.njust.edu.cn

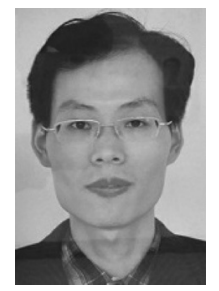

LI Yinya was born in 1976. He received his B.S. degree from Jimei University in 2001, and Ph.D. degree from Nanjing University of Science and Technology in 2006. He has been an associate professor with School of Automation, Nanjing University of Science and Technology, since 2006. His major research interests are nonlinear filtering and bearingsonly tracking.

E-mail: liyinya@mail.njust.edu.cn

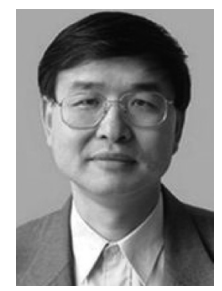

SHENG Andong was born in 1964. He received his B.S. degree and Ph.D. degree from Harbin Institute of Technology in 1985 and 1990 respectively. He has been a professor with School of Automation, Nanjing University of Science and Technology, since 1999. His major research interests are multi-agent system and distributed estimation.

E-mail: shengandong@mail.njust.edu.cn 